\title{
Individualisation thérapeutique et analgésiques : vers une médecine personnalisée
}

\author{
Individualised therapy and analgesics: moving towards personalised medicine
}

\author{
J. Desmeules $\cdot$ F. Libert $\cdot$ A. Eschalier \\ (C) Springer-Verlag France 2013
}

Au cours des dernières décennies, le développement de la biologie moléculaire et de la connaissance autour des modulateurs génétiques de la douleur rapproche le thérapeute d'un ajustement thérapeutique individualisé intégrant les caractéristiques génétiques du patient dans sa prise en charge.

En effet, il en va ainsi déjà dans certains domaines de l'oncologie où le choix du traitement ou l'ajustement de la dose peut se faire en tenant compte du bagage génétique de l'individu et/ou du tissu tumoral cible du patient. Ce « makeup génétique » permet d'affiner le choix thérapeutique et est associé à une meilleure réponse au traitement. Il en va également ainsi dans le domaine des maladies infectieuses ou dans le domaine de la neurologie où la toxicité dermatologique de médicaments comme l'abacavir ou la carbamazépine pourrait être évitée en écartant systématiquement les individus qui présenteraient une susceptibilité HLA particulière. Le domaine cardiovasculaire, en particulier de l'anticoagulation orale, est également l'objet de tests pharmacogénétiques qui permettent d'affiner le traitement sur la base d'algorithmes qui permettent d'affiner la posologie et qui sont approuvés par certaines autorités d'enregistrement.

Dans le domaine de la douleur, l'individualisation de la thérapeutique symptomatique s'inspire essentiellement de l'origine (nociceptive ou neuropathique) de la douleur et/ ou de son intensité. Cette appréciation clinique guide l'option thérapeutique symptomatique et en particulier la sélection de la classe d'analgésique à laquelle on fait appel. Ce choix est généralement suivi d'une adaptation individuelle empirique de la posologie de l'analgésique sélectionné jusqu'à l'obtention des objectifs cliniques escomptés.

\section{J. Desmeules}

Service de pharmacologie et toxicologie cliniques, centre multidisciplinaire de la douleur, hôpitaux universitaires de Genève, université de Genève, Suisse

F. Libert $(\bowtie) \cdot$ A. Eschalier

Service de pharmacologie, CHU de Clermont-Ferrand, F-63003

Clermont-Ferrand, France

e-mail : flibert@chu-clermontferrand.fr
Il arrive cependant que, pour des raisons diverses, une proportion significative de patients éprouve très précocement des effets indésirables, et ce, à faibles doses alors que d'autres, à l'autre extrême, " résistent » et ont une analgésie insuffisante malgré des doses croissantes d'analgésiques. Ainsi, l'hétérogénéité de la réponse aux médicaments analgésiques peut être en partie liée à certains facteurs constitutifs de l'individu ou environnementaux connus pour influencer la réponse individuelle aux traitements médicamenteux. D'autres comme l'âge, le sexe, le poids corporel, le tabac, l'alcool et les maladies concomitantes (notamment atteinte rénale ou hépatique) influent également sur cette variabilité.

Dans un contexte de douleur chronique, l'observance thérapeutique explique à elle seule au moins $50 \%$ de la variabilité de la réponse au traitement, mais d'autres facteurs constitutifs interviennent aussi dans l'expression de cette variabilité. Les facteurs individuels génétiques comme certains polymorphismes contribuent ainsi également à la variabilité de la réponse aux analgésiques observée et sont parmi les facteurs les plus stables et prédictifs de la variation interindividuelle de la réponse à un analgésique.

Lors du traitement de la douleur, après avoir consommé l'analgésique, celui-ci est absorbé, métabolisé, distribué à son site d'action où il interagit avec des récepteurs ou des enzymes et est finalement excrété.

Chacune de ces étapes est tributaire de facteurs génétiques bien caractérisés qui vont influencer le devenir du médicament dans l'organisme et, par voie de conséquence, la réponse au traitement.

Dans ce numéro consacré à la pharmacogénétique, nous allons considérer l'avancée des connaissances sur quelques exemples illustrant l'influence de la génétique sur différentes classes thérapeutiques antalgiques largement utilisées comme les AINS (Rollason et al.), les opioïdes (Maroussi et al.) ou les antidépresseurs (Rodieux et al.). Nous verrons également que l'insuffisance hépatocellulaire que l'on rencontre lors d'une cirrhose hépatique se rapproche phénotypiquement des métaboliseurs lents génotypiques et que des facteurs environnementaux (interactions médicamenteuses, 
déficiences d'organes) peuvent ainsi transformer un bon métaboliseur génotypique en mauvais métaboliseur phénotypique (Bosilkovska et al.). On peut à l'heure actuelle dépister par le biais de la biologie moléculaire les mutations ou les polymorphismes qui pourraient conduire à une altération de la fonction, mais on peut également aisément, par des tests validés, évaluer simplement la fonction de certains transporteurs et cytochromes P-450. L'indication de l'usage de l'un ou de l'autre est revue dans l'article de Samer et al. Par ailleurs, compte tenu de la problématique actuelle liée à l'élargissement de l'utilisation des opioïdes dans les douleurs non cancéreuses, il nous est paru important d'évaluer la composante pharmacogénétique de la dépendance à ces médicaments (Roche-Xavier et al.). Enfin, les régulations épigénétiques, qui modulent l'expression des gènes neuronaux sans affecter la séquence de l'ADN, semblent largement impliquées dans certains phénomènes de transition de la douleur aiguë à la douleur chronique. Leur compréhension amènera sans doute à une plus grande prédiction du risque de chronicisation de la douleur, et au développement de nouvelles molécules permettant d'atténuer ce phénomène (Pereira et al.).

L'avenir de la pharmacogénétique s'appuiera sur des études prospectives randomisées contrôlées et stratifiées pour les aspects génétiques. Cette approche identifiera l'influence des polymorphismes sur la réponse thérapeutique et permettra de définir le nombre de patients à génotyper pour s'épargner des effets indésirables et/ou pour améliorer l'efficacité thérapeutique. Ces travaux pourront concerner les antalgiques disponibles, mais également les nouvelles molécules. Les implications éthiques, financières et médicales de la génétique prédictive devraient être appréhendées et clairement définies pour chacun des tests. Et l'emploi du génotypage prédictif devra se limiter aux indications ayant démontré l'intérêt de la pharmacogénétique dans la prescription individualisée. 